Debate / Controversy

\title{
Reflexiones sobre la accesibilidad de la población extranjera a los programas nacionales de rentas mínimas: una lectura comparada
}

Reflections on the accessibility of the foreign population in the national minimum income programs: a comparative approach

\author{
Lluís Francesc Peris-Cancio (iD \\ Sapienza Università di Roma, Italia \\ lluisfrancesc.periscancio@uniroma1.it
}

Recibido / Received: 22/09/2020

Aceptado / Accepted: 17/01/2021

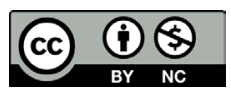

\section{RESUMEN}

El artículo propone un análisis sobre la accesibilidad de los extranjeros a los programas de renta mínima con la voluntad de estimular el debate sobre el impacto que la reciente introducción del Ingreso Mínimo Vital (IMV) puede tener en los ciudadanos de otras nacionalidades en España. Se realiza una comparación entre Alemania, Francia, Italia y España, con la finalidad de conocer las diferencias y similitudes en las condiciones de vida, el riesgo de pobreza, la exclusión social y el acceso efectivo a los regímenes de renta minima entre las poblaciones nacionales y extranjeras. En la parte conclusiva se ilustran algunos factores clave para ampliar la inclusividad de los programas observando el caso español.

Palabras clave: ingreso mínimo vital, programas de renta mínima, pobreza, migraciones.

\section{ABSTRACT}

The article proposes an analysis on the expectations concerning the accessibility of foreign people to Spanish minimum income programs and intends to fuel the debate on the impacts that the latest introduction of the Ingreso Minimo Vital (Minimum Living Income) may have on foreign citizens living in Spain. A cross-country comparison among Germany, France, Italy and Spain has been carried out in order to ascertain differences and similarities in living conditions, poverty risks, social exclusion and effective accesses to minimum income schemes among native and foreign populations in the four selected countries. Some key factors to enlarge the inclusiveness of minimum incomes schemes in Spain result from the outcomes.

Keywords: Minimum living income, minimum income programs, poverty, migrations. 
Reflexiones sobre la accesibilidad de la población extranjera a los programas nacionales de rentas mínimas: una lectura comparada

\section{INMIGRACIÓN, NACIONALIDAD Y POBREZA}

Es sabido que las personas extranjeras ocupan con frecuencia el percentil más bajo de retribución salarial (Reysz, 2016) y que se enfrentan a menudo con situaciones de subempleo (Heath y Cheung, 2007; Kogan, 2007; Van Tubergen, 2006). Además, la brecha de pobreza entre nacionales y extranjeros es todavía significativamente alta en muchos países europeos (Kesler, 2015). La inclusión de las familias extranjeras en los esquemas de ingresos mínimos genera un encendido debate político, instigado, a menudo, por minorías que se activan para fomentar una visión de las personas inmigradas como individuos que inmerecidamente se benefician del sistema de bienestar nacional.

El presente artículo ofrece un panorama de la condición de pobreza de las familias extranjeras presentes en el Estado español, aportando datos comparados que permiten valorar el impacto que la introducción del Ingreso Mínimo Vital (IMV) puede significar para la superación del estado de pobreza y/o exclusión.

Para ello, se compara la experiencia española con otros tres contextos nacionales (Alemania, Francia e Italia) elegidos en base a cuatro criterios: la relevancia de la presencia de población extranjera, la comparabilidad de los datos socioeconómicos, unos valores próximos de MIPEX $^{1}$ y la introducción reciente de experiencias nacionales de rentas mínimas.

Para una operacionalización más fácil del tratamiento de la inmigración en cada contexto se hace referencia de manera exclusiva al dato de la población con nacionalidad extranjera, renunciando a otras variables más descriptivas como el tiempo de residencia y el lugar de nacimiento. Esta simplificación permite una comparación más eficaz entre contextos nacionales en base a las elaboraciones estadísticas estatales de los programas de rentas mínimas; por el contrario, no permite considerar la influencia de la variable de la tasa de naturalización en cada país, dejando oculto el dato sobre los procesos de integración de migrantes que han obtenido la nacionalidad.

En cada país se analiza la presencia y las características de la población extranjera, y se describe la incidencia de la pobreza en base a cuatro indicadores: mediana de ingresos netos, tasa de hacinamiento, tasa de riesgo de pobreza y evolución de la tasa de riesgo de pobreza y exclusión (AROPE) en los últimos quince años.

Sucesivamente, y para una mejor contextualización del IMV español, se consideran algunas variables en el diseño de las medidas de lucha contra la pobreza en los tres países seleccionados: ALG II (Arbeitslosengeld II) en Alemania, el RSA (Revenu de solidarité Activé) en Francia, y el Rel (Reddito di inclusione) convertido en el año 2019 en el RdC (Reddito di Cittadinanza) en Italia, ilustrando los requisitos y procedimientos previstos para la población extranjera y el dato sobre su efectivo acceso. En base a los estudios empíricos que analizan el funcionamiento de estas experiencias, se anticipa una hipótesis del previsible impacto que el IMV podrá tener sobre la población extranjera en España y las variables que pueden ser más influentes.

\footnotetext{
1 El Migration Integration Policy Index mide las oportunidades que tienen los inmigrantes de participar dentro de la estructura productiva del país que los acoge. En el año 2019, el valor de este índice fue de 58 en el caso de Alemania e Italia, 56 en Francia y 60 en España (https://www.mipex.eu).
} 


\section{PRESENCIA DE LA POBLACIÓN EXTRANJERA EN ALEMANIA, FRANCIA, ITALIA Y ESPAÑA}

España e Italia son países que sólo en los últimos cincuenta años han pasado de registrar un saldo migratorio negativo a uno positivo. Esto comporta que la población extranjera sea relativamente más joven respecto a otros países con una historia de inmigración consolidada.

Según los datos de la Organización de las Naciones Unidas (ONU, 2019), España se sitúa como la segunda nación de las cuatro observadas con mayor porcentaje absoluto de personas extranjeras (9,7\%), tan solo superada por Alemania (11,7\%).

Tabla 1. Población extranjera 2018 (en \%)

\begin{tabular}{lcccc}
\hline & Alemania & Francia & Italia & España \\
\hline Extranjeros UE & 5,1 & 2,3 & 2,6 & $\mathbf{4 , 1}$ \\
Extranjeros no UE & 6,6 & 4,7 & 5,9 & $\mathbf{5 , 6}$ \\
Total ext. UE y no UE & 11,7 & 7 & 8,5 & $\mathbf{9 , 7}$ \\
\hline
\end{tabular}

Fuente: ONU (2019).

El dato hace referencia al número total de personas de nacionalidad extranjera con permiso de residencia, e incluye situaciones muy diversas por motivación y circunstancias; no contempla, sin embargo, el indeterminado número de personas extranjeras presentes en el territorio nacional en situación administrativa irregular, aspecto cualitativamente relevante para los planes de reducción de la pobreza.

El alto porcentaje de población extranjera se debe leer en relación con el número de naturalizaciones, que resulta variable en cada uno de los países de la UE. Se tenga en cuenta la diversidad de condiciones que en cada país se requiere para conceder la nacionalidad: residencia, renuncia a nacionalidad anterior, certificado de antecedentes penales, situación financiera, conocimiento del idioma, etc. (Wallace, 2010).

En los datos que se muestran en la tabla 2, se puede apreciar que es en España donde se produce un menor número de naturalizaciones, particularmente bajo en el caso de los inmigrados de países de la UE (0,1\%).

Tabla 2. Tasa anual de naturalización 20172 (en \%)

\begin{tabular}{lcccc}
\hline & Alemania & Francia & Italia & España \\
\hline Extranjeros UE & 1 & 0,6 & 0,7 & $\mathbf{0 , 1}$ \\
Extranjeros no UE & 1,5 & 3,4 & 3,9 & $\mathbf{2 , 6}$ \\
Total ext. UE y no UE & 2,5 & 4 & 4,6 & $\mathbf{2 , 7}$ \\
\hline
\end{tabular}

Fuente: EUROSTAT 2019 (migr_acq). Acquisition of citizenship by age group, sex and former citizenship.

La adquisición de la nacionalidad española es un camino largo y no exento de complicaciones (Dag Tjaden y Sánchez-Montijano, 2013). En su concepción, podría influir el pasado colonial, que han producido en el tiempo un trato privilegiado reservado a los

\footnotetext{
2 Tasa anual de naturalización = (Número de personas que adquieren la nacionalidad en año X/ № de extranjeros empadronados al inicio del año X) *100.
} 
Reflexiones sobre la accesibilidad de la población extranjera a los programas nacionales de rentas mínimas: una lectura comparada

ciudadanos procedentes de países que pertenecieron a la corona española (Finotelli y La Barbera, 2013).

Otro aspecto que ha caracterizado la inmigración en España es el bajo peso proporcional que tiene la presencia de refugiados respecto al total de personas extranjeras, aun cuando en los últimos años aparezca un repunte con la llegada de solicitudes provenientes de ciudadanos de Venezuela, Siria y Honduras (Consejo Económico y Social de España, 2019). Como se puede observar en la tabla 3, España no sólo recibe un bajo número de solicitudes de asilo al año (11.895 respecto a las 95.205 de Italia, 115.050 de Francia o 179.210 de Alemania) sino que, además, del total de peticiones de acogida, solo ha sido reconocido algún tipo de protección al $24 \%$ de los casos, el más bajo de los cuatro.

Tabla 3. Protección internacional 2018

\begin{tabular}{lcccc}
\hline & Alemania & Francia & Italia & España \\
\hline Solicitudes totales (applications) & 179.210 & 115.050 & 95.205 & $\mathbf{1 1 . 8 9 5}$ \\
$\begin{array}{l}\text { Reconocimiento en \% (first instance } \\
\text { decisions) }\end{array}$ & 42 & 28 & 32 & $\mathbf{2 4}$ \\
\hline
\end{tabular}

Fuente: EUROSTAT 2019 (migr_asyappctza). First-time asylum applications by citizenship, age and sex. Annual aggregated data.

Por otro lado, España es el segundo país que más órdenes de retorno realiza (59.255), con una proporción de expulsiones ejecutadas ${ }^{5}$ en línea con los demás países, con la excepción de Alemania, como se puede observar en la tabla 4.

Tabla 4. Órdenes de retorno 2018

\begin{tabular}{lcccc}
\hline & Alemania & Francia & Italia & España \\
\hline Decretadas (n. total) & 52.930 & 105.560 & 27.070 & $\mathbf{5 9 . 2 5 5}$ \\
Ejecutadas (en \%) & 55 & 15 & 21 & $\mathbf{2 0}$ \\
\hline
\end{tabular}

Fuente: EUROSTAT 2019 (migr_eiord). Third country nationals ordered to leave - annual data; EUROSTAT 2019- migr eirtn: third country nationals returned following an order to leave annual data.

Visto el cuadro general de la situación y de las dimensiones de la presencia de extranjeros en España, se requiere un análisis ulterior para valorar la situación económica en la que se encuentran y estimar lo más objetivamente posible la eventual necesidad de este conjunto de la población de beneficiarse de los programas de ingresos mínimos. Para ello se emplean específicos indicadores que describen la incidencia de las situaciones de vulnerabilidad y/o deprivación.

\footnotetext{
3 Número de personas que han presentado una primera solicitud de asilo en el país en el año 2018.

4 Se refiere al porcentaje de las decisiones en primera instancia que reconocen la concesión del estatuto de refugiado, la protección subsidiaria o una autorización de residencia temporal por circunstancias excepcionales concedida por razones humanitarias.

5 Según la directiva 2008/115/CE del Parlamento Europeo y del Consejo, se entiende por "órdenes de retorno" una decisión o acto de naturaleza administrativa o judicial por el que se declare irregular la situación de un nacional de un tercer país y se imponga o declare una obligación de retorno; asimismo, se define "expulsión" la ejecución de la obligación de retornar, es decir, el transporte físico fuera del Estado miembro.
} 


\section{POBREZA Y NACIONALIDAD EN UNA PERSPECTIVA COMPARADA}

Para analizar la incidencia de la pobreza de las personas inmigradas en los cuatro países se comparan los datos absolutos y relativos entre nacionales y extranjeros. Como se puede observar en la tabla 5, España no sólo es el país cuya población extranjera se muestra más empobrecida, sino que, además, la diferencia entre la situación entre nacionales y extranjeros es la más desigual: la mediana de ingresos netos de los residentes de terceros países es un 42,9\% menor respecto a la de los ciudadanos españoles.

Tabla 5. Mediana ingresos netos (en €)

\begin{tabular}{lcccc}
\hline & Alemania & Francia & Italia & España \\
\hline Extranjeros UE & 22.283 & 19.050 & 12.393 & 9.774 \\
Extranjeros no UE & 18.488 & 15.165 & 11.438 & 8.826 \\
Nacionales & 22.964 & 23.059 & 17.867 & 15.459 \\
Dif. Ext. no UE - Nac (\%) & $-19,3$ & $-34,2$ & $-35,9$ & $\mathbf{- 4 2 , 9}$ \\
\hline
\end{tabular}

Fuente: Tarchi et al. (2019).

Por cuanto se refiere a la vivienda, los datos muestran una diferencia generalizada entre la tasa de hacinamiento ${ }^{6}$ de nacionales y extranjeros, más pronunciada en el caso de residentes de terceros países. En el caso español alcanza un valor seis veces mayor.

Tabla 6. Tasa de hacinamiento

\begin{tabular}{lcccc}
\hline & Alemania & Francia & Italia & España \\
\hline Extranjeros UE & 15 & 15,4 & 43,9 & $\mathbf{4 , 1}$ \\
Extranjeros no UE & 16,5 & 27,5 & 55,3 & $\mathbf{1 9 , 4}$ \\
Nacionales & 6,2 & 5,9 & 22,7 & $\mathbf{3 , 4}$ \\
Dif. Ext. no UE (\%) & +266 & +466 & +243 & $\mathbf{+ 5 7 0}$ \\
\hline
\end{tabular}

Fuente: Eurostat 2019 (ilc_lvho05a). Overcrowding rate by household type - EU-SILC survey.

En base a los indicadores Eurostat EU-SILC, son seguramente significativos los datos sobre el riesgo de pobreza y/o exclusión según el indicador internacional At risk of poverty or social exclusion (AROPE), ya que el concepto emplea una concepción multidimensional de la pobreza y combina tres subindicadores: a. personas en riesgo de pobreza después de transferencias sociales, entendido como aquellas cuyos ingresos por unidad de consumo son inferiores al $60 \%$ de la renta mediana; b. carencia material severa, identificado como la proporción de la población que vive en hogares que carecen al menos de cuatro conceptos sobre nueve descritos ${ }^{7}$; y c. personas que viven en hogares con muy baja intensidad de trabajo, correspondiente a aquellas que viven en hogares en los que sus miembros en edad de trabajar lo hicieron menos del $20 \%$ de su potencial total de trabajo.

6 La tasa de hacinamiento (Household Overcrowding rate) considera que una persona vive en situación de hacinamiento si el hogar no tiene: una habitación para el hogar; una habitación por pareja en el hogar; una habitación para cada persona soltera de 18 años o más; una habitación por pareja de personas solteras del mismo sexo entre 12 y 17 años; una habitación para cada persona soltera entre 12 y 17 años y no incluida en la categoría anterior y, por último, una habitación por pareja de niños menores de 12 años.

7 Los nueve indicadores son los siguientes: no puede permitirse ir de vacaciones al menos una semana al año; no puede permitirse una comida de carne, pollo o pescado al menos cada dos días; no puede permitirse mantener la vivienda con una temperatura adecuada; no tiene capacidad para afrontar gastos imprevistos (de 650 euros); ha tenido retrasos en el pago de gastos relacionados con la vivienda principal (hipoteca o alquiler, recibos de gas, comunidad...) o en compras a plazos en los últimos 12 meses; no puede permitirse disponer de un automóvil; no puede permitirse disponer de teléfono; no puede permitirse disponer de un televisor, y, por último, no puede permitirse disponer de una lavadora. 
Reflexiones sobre la accesibilidad de la población extranjera a los programas nacionales de rentas mínimas: una lectura comparada

El gráfico 1 analiza la evolución de la tasa de riesgo pobreza y exclusión (AROPE) de la población extranjera en los últimos quince años:

Gráfico 1. Evolución de la tasa de riesgo de pobreza y exclusión (AROPE) de la población extranjera por país 2004-2019

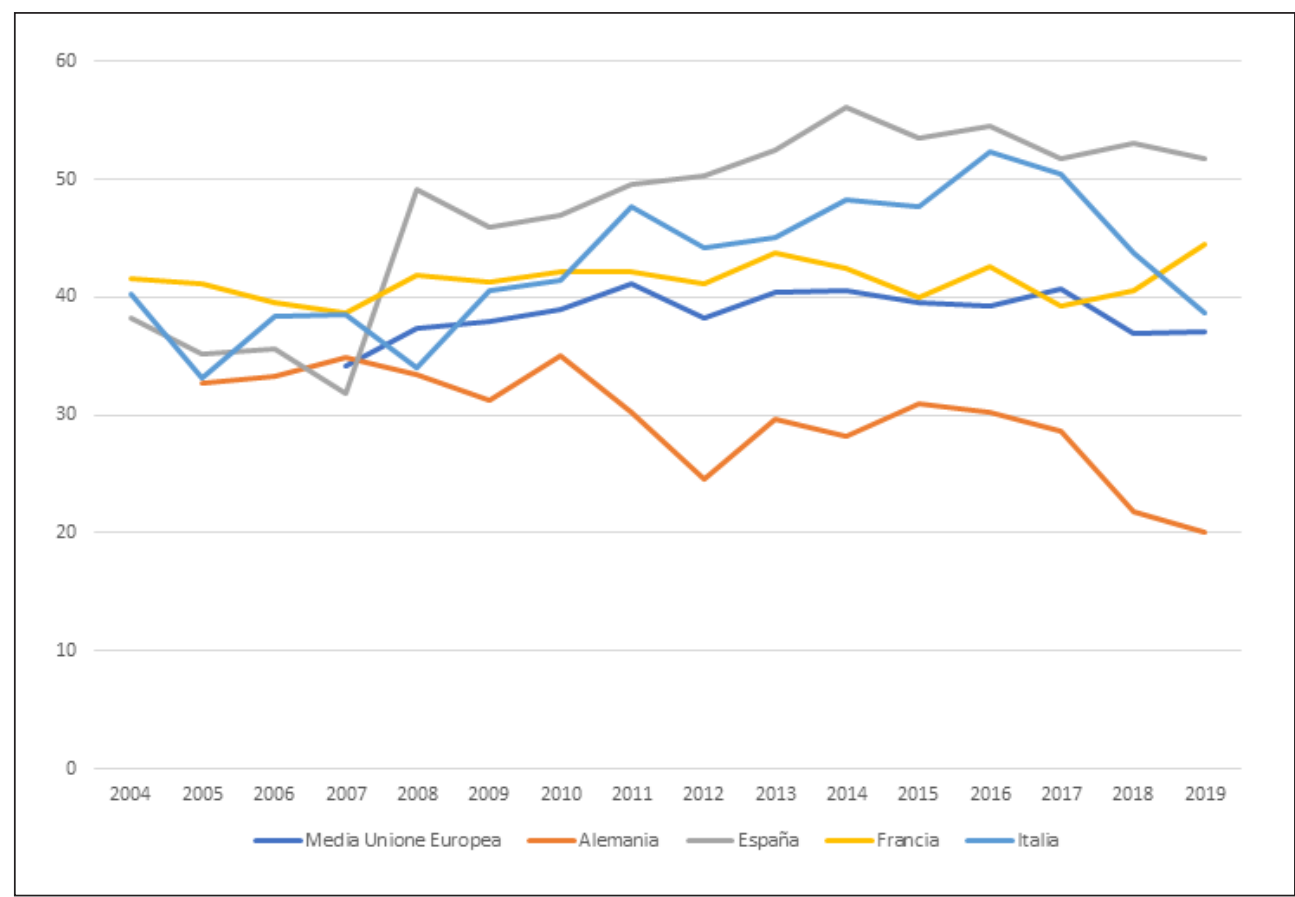

Fuente: Eurostat EU-SILC 2020 (ILC_PEPS05) People at risk of poverty or social exclusion by broad group.

Se observa que el índice AROPE de los extranjeros es alto en todos los países, con una media europea que se mantiene en torno a 40. Es interesante notar la evolución del dato en cada país, ya que se encuentran tendencias opuestas: mientras en Alemania se aprecia una progresiva reducción, España registra un aumento continuado que se estabiliza a partir del año 2012. En el caso de Francia e Italia las variaciones son menos pronunciadas.

El gráfico parece describir bien dos hechos importantes: el impacto de la crisis económica a partir del 2007, que ha significado un incremento en casi todos los casos, $y$, en segundo lugar, el posible efecto positivo que la introducción de los esquemas de rentas mínimas, con la excepción de Francia, ha supuesto en la reducción de la situación de pobreza y exclusión en todos los países, de manera más evidente en el caso italiano.

Si se compara la tasa AROPE entre nacionales y extranjeros, se aprecian también importantes diferencias entre los países estudiados (ver gráfico 2). 
Gráfico 2. Comparación de la evolución de la tasa de riesgo de pobreza y exclusión (AROPE) entre la población con nacionalidad y extranjera por país 2004-2019
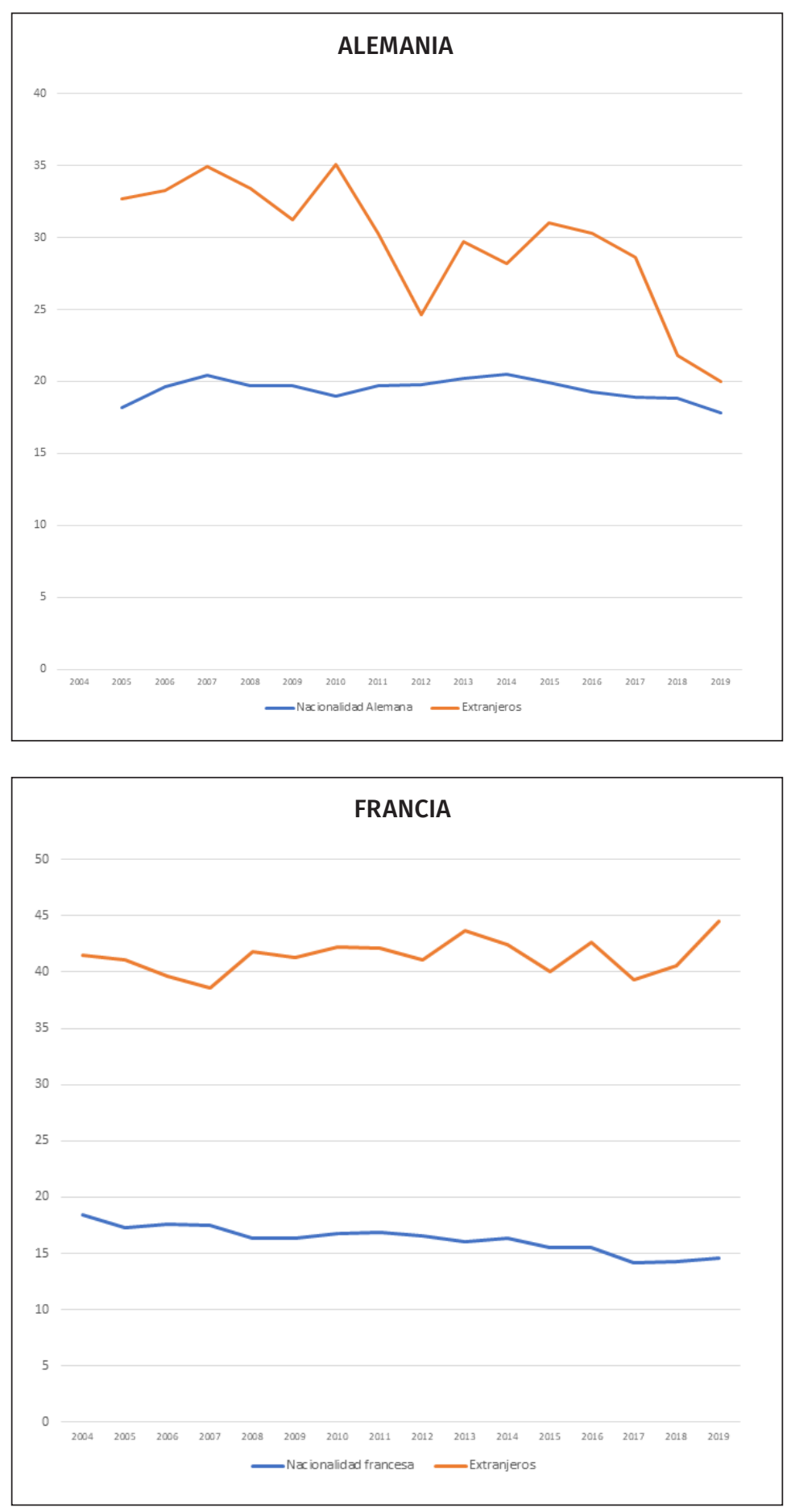
Reflexiones sobre la accesibilidad de la población extranjera a los programas nacionales de rentas mínimas: una lectura comparada
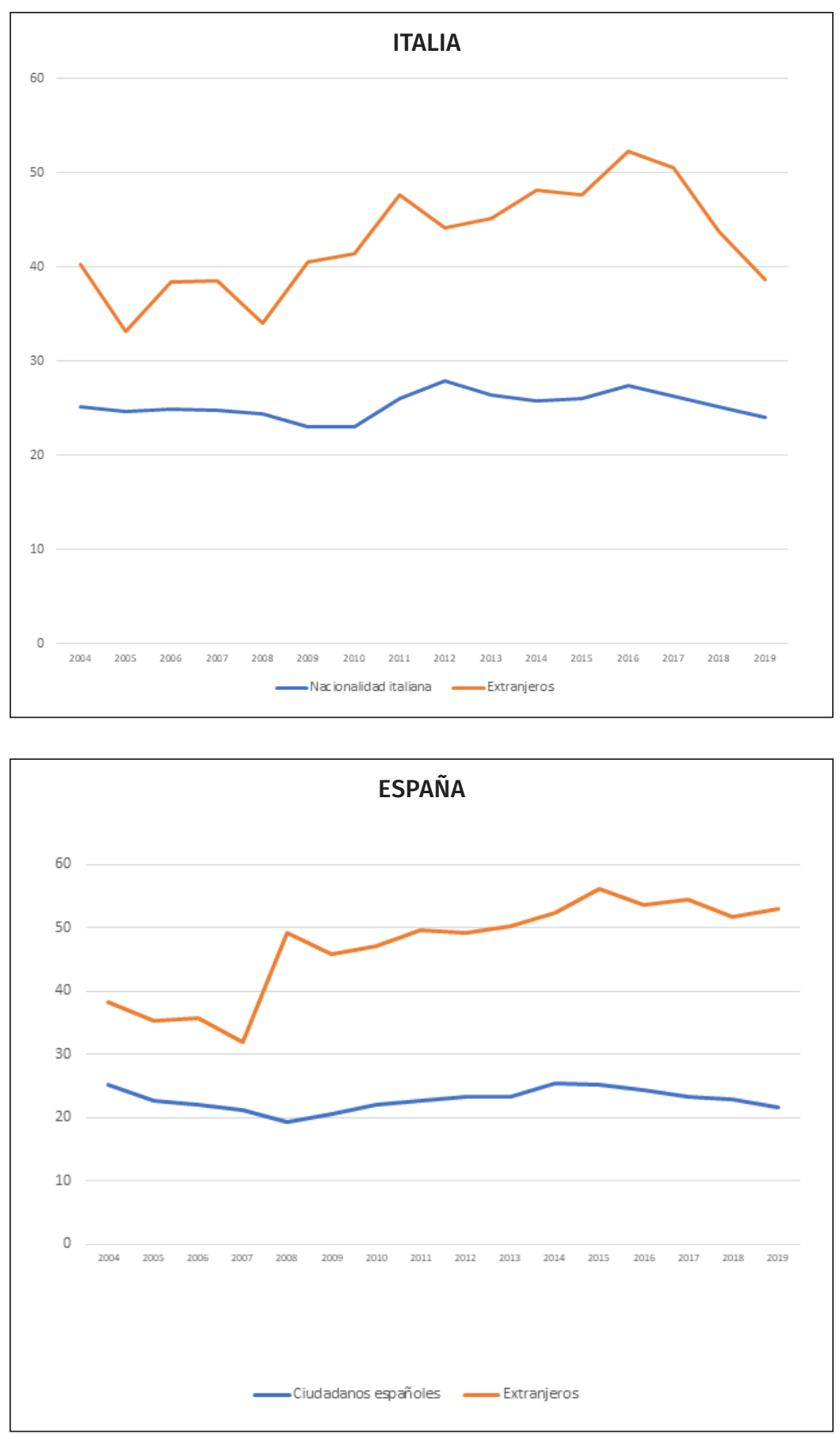

Fuente: Eurostat EU-SILC 2020 (ILC_PEPS05) People at risk of poverty or social exclusion by broad group. 
La línea del índice AROPE de la población extranjera respecto a la de los nacionales es menos estable en todos los casos. La dinámica en Alemania indica una evolución que tiende claramente a una reducción de las diferencias entre ambos grupos que alcanza en el año 2019 una distancia de sólo 2,2 puntos. El caso francés muestra una brecha consolidada e inmutable de grandes dimensiones que se perpetua durante los quince años. La realidad italiana describe un progresivo incremento de la diferencia que cambia de tendencia en los últimos años, periodo que coincide con la introducción los programas de rentas mínimas.

En el caso español, hasta el colapso de la economía en el año 2007, aparece una tendencia decreciente de la pobreza y la exclusión tanto en nacionales como en extranjeros; sin embargo, en los dos años sucesivos a la crisis, se registra un aumento imponente de la situación de pobreza en los residentes comunitarios y de terceros países, propensión que continuará en modo menos acelerado hasta nuestros días. Según el informe de la European Anti Poverty Network (2020), el detalle del dato indica que es menor en la población extranjera procedente de países de la Unión Europea, que soporta tasas muy inferiores a las personas provenientes de África y Latinoamérica.

No es posible medir la incidencia precisa que los programas de rentas mínimas hayan podido tener en contener o modificar la tendencia de estas dos líneas de pobreza y/o exclusión, y establecer cuánto hayan podido ser determinantes para una mayor convergencia o divergencia en la situación de nacionales y extranjeros; en cualquier caso, es útil analizar las experiencias y recoger los resultados de los estudios que han tratado esta cuestión en otros contextos nacionales.

\section{ESQUEMAS DE RENTAS MÍNIMAS EN EUROPA Y MECANISMOS INFLUYENTES EN LA ACCESIBILIDAD DE LA POBLACIÓN EXTRANJERA}

Las distintas leyes de institución de los sistemas de rentas mínimas en Europa adquieren características particulares según las variables de duración, perfil de los beneficiarios, condicionalidad y obligaciones, importe del beneficio, compatibilidad con una eventual actividad laboral, etc., como también las experiencias de las leyes autonómicas en España muestran diversidad (Ministerio de Sanidad, Consumo y Bienestar Social, 2018; Autoridad Independiente de Responsabilidad Fiscal, 2019). La tabla 7 ilustra algunos aspectos descriptivos en los cuatro países de referencia.

Tabla 7. Esquemas de rentas mínimas

\begin{tabular}{lllll}
\hline & Alemania & \multicolumn{1}{c}{ Francia } & Italia & España \\
\hline $\begin{array}{l}\text { Cuantía mensual para hogares } \\
\text { unipersonales (en } € \text { ) }\end{array}$ & 432 & 565 & 500 & 462 \\
$\begin{array}{l}\text { Para unidades de convivencia de } \\
\text { cuatro miembros (en } € \text { ) }\end{array}$ & 1311 & 1186 & 900 & 877 \\
$\begin{array}{l}\text { Duración } \\
\text { Complemento ayuda alquiler }\end{array}$ & Sin límite & Sin límite. & 18 meses & Sin límite \\
Edad & No & Sí & Sí & Sí \\
Requisito tiempo de residencia & $\geq 15$ & $\geq 25$ & $\geq 18$ & $\geq 23$ \\
\hline
\end{tabular}

Fuente: European Commission (2020c).

8 Prevista su introducción con un reglamento específico.

9 Tanto en el caso francés como en el español se ofrece el dato general, pero existen excepciones para personas de menor edad de la indicada con hijos a cargo y/o otras circunstancias subjetivas. 
Reflexiones sobre la accesibilidad de la población extranjera a los programas nacionales de rentas mínimas: una lectura comparada

La renta mínima en Alemania se basa en la condicionalidad: para poder acceder al derecho, las personas deben ofrecer la disponibilidad para trabajar al menos tres horas al día. La edad de los beneficiarios parte desde los quince años hasta la edad legal de jubilación (European Commission, 2020a). Aunque se llama beneficio por desempleo, es posible continuar con el programa ALG II mientras se obtiene un ingreso por trabajo que no alcance el coste mínimo de la vida. Los extranjeros deben residir legalmente en Alemania al menos tres meses antes de realizar la solicitud y demostrar haber tenido con anterioridad una experiencia laboral. Los ciudadanos no pertenecientes a la UE están obligados además a poseer un permiso de residencia que les permita trabajar (Bundesagentur für Arbeit, 2011). Todos los beneficiarios están sujetos a la participación en medidas de integración orientadas a la inserción laboral.

Desde que en el año 2009 se introdujo en Francia el Revenu de solidarité active (RSA) se concibe como un ingreso que excluye a los menores de 25 años sin hijos dependientes y a los estudiantes. Los requisitos para acceder al beneficio de la RSA prevén que los beneficiarios europeos que no tienen ciudadanía francesa deban haber vivido en Francia durante al menos tres meses antes de la solicitud. Además, los extranjeros de terceros países deben poseer un permiso de residencia con el que justifiquen haber trabajado en Francia durante al menos cinco años antes de su solicitud (European Commission, 2020b), aspecto que excluye a un número consistente de extranjeros.

A diferencia del caso alemán y francés, el Reddito di Inclusione (REI) se introdujo mucho más tarde, en marzo del 2017. Hasta el REI, Italia no tenía ningún programa de ingresos mínimos garantizados a nivel nacional, sólo a través de los gobiernos regionales con diversos esquemas y modalidades de aplicación (Sgritta, 2020). Con el cambio de ejecutivo, el Reddito di Inclusione fue reemplazado en abril de 2019 por una nueva medida, el Reddito di Cittadinanza, que se concibe como un ingreso mínimo más generoso y de mayor duración, pero no permanente. Observa también reglas más estrictas de condicionalidad si se compara con el Rel (Jessoula, Natili y Raitano, 2019). El requisito de la residenza rinforzata es el que más excluye a la población extranjera, ya que significa la obligación de demostrar haber sido residente en Italia diez años.

En los tres contextos nacionales estudiados, la proporción de beneficiarios extranjeros es alto; en Alemania, el $20 \%$ de los beneficiarios del Arbeitslosengeld II en el año 2010 eran extranjeros (Riphahn, Sander, Bamberg y Wunder, 2010) en comparación con una cuota sobre la población próxima al 11,7\%. En 2018, según la Agencia federal alemana de Empleo (Bundesagentur für Arbeit), el 55\% de los beneficiarios de ALG II tenían un pasado migratorio, es decir, personas con al menos uno de los padres nacidos con ciudadanía no alemana (Brenke, 2010). En Francia, de los 1,88 millones de beneficiarios del Revenu de solidarité Activé en diciembre de 2017 , el $16 \%$ eran extranjeros, en comparación con el $7 \%$ de la cuota total sobre la población (Direction de la recherche, des études, de l'évaluation et des statistiques [DREES], 2019).

En Italia, entre enero de 2018 y marzo de 2019, el porcentaje total de los ciudadanos no pertenecientes a la UE beneficiarios del Rel fue del 11\% (Istituto Nazionale Previdenza Sociale [INPS], 2019), una cifra próxima al 8,5\% de la incidencia de los extranjeros sobre la población total.

Según Brenke (2010), la participación comparativamente elevada de los extranjeros en el plan ALG II alemán no siempre trajo consigo como resultado la reducción directamente proporcional de la pobreza de este colectivo. Su estudio demuestra un efecto no deseado por el cual, aquellos con una situación más acentuada de pobreza, encuentran mayor dificultad para acceder al beneficio a causa de determinados obstáculos administrativos. También se ha descrito que el beneficio económico que prevé este instrumento de renta 
mínima podría llegar a influir negativamente en la consecución de los requisitos necesarios para obtener un permiso de residencia de larga duración, desincentivando a participar en el programa a aquellos que no lo poseen (Riphahn et al., 2010).

Tabla 8. Inmigrantes y participación en programas nacionales de rentas mínimas en Alemania, Francia e Italia. Año 2018

\begin{tabular}{lcccc}
\hline & Alemania & Francia & Italia & España \\
\hline $\begin{array}{l}\text { Población extranjera presente en el país } \\
\text { (\%) }\end{array}$ & 11,7 & 7 & 8,5 & $\mathbf{9 , 7}$ \\
$\begin{array}{l}\text { Población extranjera en riesgo de pobreza } \\
\text { (no UE, en \%) }\end{array}$ & 23,6 & 50,1 & 45,7 & $\mathbf{5 6 , 5}$ \\
$\begin{array}{l}\text { Población extranjera beneficiaria renta } \\
\text { básica (\%) }\end{array}$ & $20(1)$ & $16(2)$ & $11(3)$ & *(4) \\
\hline
\end{tabular}

Fuente: elaboración del autor sobre datos Eurostat, 2019

Fuente: Riphahn et al. (2010), datos referidos al año 2010.

Fuente: DREES (2019), datos referidos al 2017.

Fuente: INPS (2019), datos de enero 2018 a marzo 2019.

Datos no disponibles.

Según Brenke (2010), la participación comparativamente elevada de los extranjeros en el plan ALG II alemán no siempre trajo consigo como resultado la reducción directamente proporcional de la pobreza de este colectivo. Su estudio demuestra un efecto no deseado por el cual, aquellos con una situación más acentuada de pobreza, encuentran mayor dificultad para acceder al beneficio a causa de determinados obstáculos administrativos. También se ha descrito que el beneficio económico que prevé este instrumento de renta mínima podría llegar a influir negativamente en la consecución de los requisitos necesarios para obtener un permiso de residencia de larga duración, desincentivando a participar en el programa a aquellos que no lo poseen (Riphahn et al., 2010).

En el caso francés se ha observado que, a parte de los problemas de accesibilidad que afectan tanto a la población francesa como a la extranjera, la mayoría de los inmigrantes nacidos fuera de Europa no pudieron demostrar un permiso de residencia de trabajo en Francia de más de cinco años precedente a la solicitud del beneficio. Existe también una dificultad para la participación de los extranjeros al programa ligada a la imposibilidad de poder aceptar propuestas laborales de baja calidad (European Minimum Income Network, 2014). Otro elemento crítico es que las mujeres que entraron en Francia a través de la reunificación familiar tuvieron mayor dificultad de acceso, ya que no podían demostrar el haber pasado al menos cinco años en el país (Organización para la Cooperación y el Desarrollo Económicos [OECD], 2018). Por su parte Sainsbury (2012) considera negativo el efecto del establecimiento de una edad mínima de 25 años, ya que comporta la exclusión de una buena parte de la población juvenil en situación de necesidad, en gran medida con un pasado migratorio. El autor registra también la menor capacidad de atención del ente local hacia los extranjeros, con la evidencia empírica de que el acompañamiento profesional tiene una duración media menor en la atención a ciudadanos nacidos en otros países que con nacionales al no poseer adecuados servicios profesionales de mediación lingüística y cultural.

La experiencia en Italia tiene menos recorrido. Se estima que alrededor de la mitad de los extranjeros en situación de pobreza no pidieron o no recibieron el Rel por diversos motivos (OECD, 2019). Por un lado, estaban obligados a haber permanecido en Italia durante al menos dos años con un permiso de residencia (sorprendentemente aumentado a diez 
Reflexiones sobre la accesibilidad de la población extranjera a los programas nacionales de rentas mínimas: una lectura comparada

años con el sucesivo Reddito di Cittadinanza); por otro, los procedimientos burocráticos que tienen que ver con la obligación de un certificado ISEE $^{10}$ dificultan el acceso de los extranjeros a los programas, especialmente si no pueden beneficiarse de apoyo lingüístico y de traducción.

Todas las observaciones anteriores están al origen de una menor participación de la población extranjera, como manifestación de un efecto no deseado en el diseño de los esquemas de rentas mínimas.

\section{REFLEXIONES CONCLUSIVAS}

El panorama descrito permite observar la complejidad del tratamiento objeto de esta reflexión. A modo de conclusión, se puede afirmar que la facilitación del acceso a la participación de las personas extranjeras es un tema central en la futura evaluación del IMV: en primer lugar, por su consistente presencia demográfica, $y$, en segundo, porque, respecto a los otros tres contextos nacionales estudiados, España se presenta como un país en el que las personas y familias extranjeras se encuentran en una situación de mayor pobreza y/o exclusión social.

En espera de lo primeros datos ciertos en las memorias de actuación del IMV, se puede inferir, partiendo de la experiencia de otros países, que este reto depende, entre otros, de dos cuestiones principales: las características del esquema de la renta básica y la inversión en su implementación.

Por lo que respecta al primer punto, el IMV ofrece características que son facilitadoras de la inclusión de los extranjeros respecto a los cuatro esquemas de rentas mínimas descritos: no tiene un límite temporal, no aplica condicionalidad a las unidades de convivencia que benefician de la ayuda, permite una inserción progresiva en el mundo laboral compatible con la prestación, pone una atención a las víctimas de trata de seres humanos y explotación sexual, y no está vinculada a una precedente experiencia laboral; podrían ser más inclusivos el requisito de un año de residencia y la edad mínima de veintitrés años. En cualquier caso, el cuadro es más bien favorable desde este punto de vista y concede un cierto optimismo.

Menos clara aparece la cuestión de su implementación. Seguramente es pronto para hacer valoraciones, más aún en el contexto de emergencia sanitaria en el que se ha gestado su nacimiento; con todo, aparece indispensable la realización de un esfuerzo en la simplificación de todo el procedimiento de solicitud y gestión.

El punto clave será la governance de su arquitectura institucional multilevel y la implicación de los distintos niveles de la administración pública. El IMV abre la puerta a una gestión descentralizada a través de la modalidad de convenio o, en el caso de Navarra y País Vasco, de la asunción directa de las funciones de las comunidades autónomas, pero no impone una obligatoriedad general de participación autonómica en la administración de la prestación. De esta forma, es previsible que algunas autonomías renuncien a gestionar el IMV, otras participen sin añadir mejoras y un tercer grupo participe desarrollando la política de modo complementario a distintos niveles: con el aumento de las cuantías, con la cobertura de nuevos colectivos (como podrían ser los extranjeros en situación irregular), con el desarrollo de la información y la proactividad en la inclusión de beneficiarios, con un acompañamiento técnico de las unidades de convivencia mediante acciones de promoción social (servicios sociales, empleo, mediación cultural), etc.

\footnotetext{
10 El ISEE (Indicatore della situazione Economica Equivalente) es un instrumento que define con un índice la situación económica de las familias según diferentes variables; se usa a menudo como elemento para demostrar la necesidad de ayudas económicas o de reducciones en el coste de servicios públicos.
} 
Por ello, en el futuro, junto con los datos generales, será necesario hacer una evaluación por territorios, esperando que, come parece lógico temer, no se perpetúen las diferencias regionales hoy existentes, también en el acceso de las personas extranjeras, sino que se diseminen las mejores prácticas hoy existentes.

\section{REFERENCIAS}

Autoridad Independiente de Responsabilidad Fiscal (2019). Los programas de rentas mínimas en España. Estudio. Madrid: AlReF.

Brenke, K. (2010). Five Years after the Reform of the Social and Unemployment Benefits in Germany. Weekly Report, Deutsches Institut für Wirtschaftsforschung, 6(12), 86-96.

Bundesagentur für Arbeit (2011). Basic Social Security for Job Seekers. Arbeitslosengeld II (Social Security). Recuperado de

https://www.kreis-lup.de/export/sites/LUP/.galleries/PDF-LUP1/PDF-FD16/ Ausfuellhinweise_Merkblaetter-ALG-II-mehrsprachig/Merkblatt-Sozialgesetz-ALG-IIenglisch.pdf

Consejo Económico y Social de España (2019). Informe 02/2019. La inmigración en España: efectos y oportunidades. Madrid: Consejo Económico y Social.

Dag Tjaden, J. y Sánchez-Montijano, E. (2013). El acceso a la ciudadanía y sus efectos sobre la integración de inmigrantes: Manual para España. Bruselas: MPG.

Direction de la recherche, des études, de l'évaluation et des statistiques (2019). La protection sociale en France et en Europe en 2017. Résultats des comptes de la protection sociale. Recuperado de

https://drees.solidarites-sante.gouv.fr/publications/panoramas-de-la-drees/laprotection-sociale-en-france-et-en-europe-en-2017-resultats

European Anti Poverty Network (2020). El estado de la pobreza. Seguimiento del indicador de pobreza y exclusión social en España 2008-2019. Madrid: EAPN.

European Commission (2020a). Germany - Unemployment benefits. Employment, Social Affairs \& Inclusion. Recuperado de https://ec.europa.eu/social/main.jsp?catld=1111\&langld=en\&intPageld=4557

European Commission (2020b). France - Income Support (RSA). Employment, Social Affairs \& Inclusion. Recuperado de https://ec.europa.eu/social/main.jsp?catld=1110\&langld=en\&intPageld=4541

European Commission (2020c). Employment, Social Affairs \& Inclusion, Social Protection \& Social Inclusion, Brussels.

European Minimum Income Network (2014). Analysis of Welfare Benefits Systems: The case of the "Revenu de solidarité active" in France. Recuperado de https://eminnetwork.files.wordpress.com/2013/04/emin-france-2014-en.pdf

EUROSTAT (2019). European Statitistical Recovery Dashboard; tables by themes, population and social conditions database. Luxemburg.

EUROSTAT (2020). Income and living conditions database. Luxemburg.

Finotelli, C. y La Barbera, M. C. (2013). When the exception becomes the rule: The Spanish citizenship regime. Migration Letters, 10, 245-253. 
Reflexiones sobre la accesibilidad de la población extranjera a los programas nacionales de rentas mínimas: una lectura comparada

Heath, A. y Cheung, S. Y. (2007). Unequal Chances: Ethnic Minorities in Western Labour Markets. Oxford: Oxford University Press.

Istituto Nazionale Previdenza Sociale (2019). Osservatorio sul Reddito e Pensione di Cittadinanza. Appendice di statistica. Roma: INPS.

Jessoula, M., Natili, M. y Raitano, M. (2019). Italy: Implementing the new minimum income scheme. ESPN Flash Report. Recuperado de https://ec.europa.eu/social/BlobServlet?docld=21476\&langld=en

Kesler, C. (2015). Welfare states and immigrant poverty: Germany, Sweden, and the United Kingdom in comparative perspective. Acta Sociológica, 58(1), 39-61. https://doi.org/10.1177/0001699314560238

Kogan, I. (2007). Working Through Barriers: Host Country Institutions and Immigrant Labour Market Performance in Europe. Dordrecht: Springer.

Ministerio de Sanidad, Consumo y Bienestar Social (2018). El Sistema Público de Servicios Sociales. Informe de rentas mínimas de inserción: año 2018. Madrid: Ministerio de Sanidad, Consumo y Bienestar Social

Organización de las Naciones Unidas (2019). International Migrant Stock 2019. Recuperado de https://www.un.org/en/development/desa/population/migration/data/estimates2/ estimates19.asp

Organización para la Cooperación y el Desarrollo Económicos (2018). Working Together for Local Integration of Migrants and Refugees in Paris. París: OECD Publishing.

Organización para la Cooperación y el Desarrollo Económicos (2019). Working Together for Local Integration of Migrants and Refugees in Rome. París: OECD Publishing.

Reysz, J. (2016). The return to work policies in a time of crisis: A comparison between Germany and France. Recuperado de https://ideas.repec.org/p/hal/journl/halshs-01568258.html

Riphahn, R. T., Sander, M., Bamberg, U. y Wunder, C. (2010). The welfare use of immigrants and natives in Germany: The case of Turkish immigrants. International Journal of Manpower, 34(1), 70-82.

Sainsbury, D. (2012). Welfare States and Immigrant Rights: The Politics of Inclusion and Exclusion. Oxford: Oxford University Press.

Sgritta, G. (2020). Politiche e misure della povertà: il reddito di cittadinanza. Politiche Sociali/Social Policies, 1, 39-56.

Tarchi, D., Sermi, F., Belmonte, M., Mcmahon, S., Kalantaryan, S. y Gilodi, A. (2019). Atlas of Migration - 2019. Luxemburgo: Publications Office of the European Union.

Van Tubergen, F. (2006). Immigrant Integration: A Cross-national Study. Nueva York: LFB Scholarly Publishing.

Wallace, S. (2010). Naturalization policies in Europe: Exploring patterns of inclusion and exclusion. Florencia: EUDO Citizenship Observatory/Robert Schuman Center for Advanced Studies. 